\title{
User Needs Analysis and Development of Priorities for Life Cycle Impact Assessment
}

\author{
Mary Stewart ${ }^{1}$ and Olivier Jolliet ${ }^{2 *}$ \\ ${ }^{1}$ Department of Chemical Engineering, University of Sydney, Sydney, NSW, 2006, Australia \\ 2 Industrial Ecology \& Life Cycle Systems Group, GECOS-Institute of Environmental Sciences and Technology, \\ Ecole Polytechnique Fédérale de Lausanne (EPFL), CH-1015 Lausanne, Switzerland
}

* Corresponding author (olivier.jollier@epfl.ch)

With contributions from Jane Bare, Alan Brent, Mark Goedkoop, Norihiro Itsubo, Ruedi Mueller-Wenk, Claudia Peña, Gerald Rebitzer, Rita Schenk, Helias Udo de Haes and Bo Weidema

\begin{abstract}
The development of the LCIA programme of the UNEP/SETAC Life Cycle Initiative started with a global survey of LCA practitioners. There were $91 \mathrm{LCIA}$-specific responses from all global regions. Respondents gave an indication of how they use LCA with respect to both the stage of LCA that they base decisions on (LCI, LCIA or a combination of both) as well as the types of decisions which they support with LCA information. The issues requiring immediate attention within the UNEP SETAC Life Cycle Initiative identified from this User Needs analysis are the need for transparency in the methodology, for scientific confidence and for scientific co-operation as well as the development of a recommended set of factors and methodologies. Of interest is the fact that results from the different regions highlighted the need for different impact categories. Based on this information proposals were made for new impact categories to be included in LCA (and thus LCIA).

The LCIA programme aims to enhance the availability of sound LCA data and methods and to deliver guidance on their use. More specifically, it aims to 1) make results and recommendations widely available for users through the creation of a worldwide accessible information system and 2) establish recommended characterisation factors and related methodologies for the different impact categories, possibly consisting of sets at both midpoint and damage level. The work of the LCIA programme of the UNEP/SETAC Life Cycle Initiative has been started within four task forces on 1) LCIA information system and framework, 2) natural resources and land use, 3) toxic impacts, and 4) transboundary impacts. All participants willing to contribute to these efforts are invited to contact the LCIA programme manager or to join the next LCIA workgroup meeting that will take place in at the world SETAC congress in Portland on Thursday 18 November 2004.
\end{abstract}

\section{Introduction}

The Life Cycle Impact Assessment (LCIA) Programme is one of the three programmes of the UNEP/SETAC Life Cycle Initiative. The over-arching aim of this programme is to enhance the availability of sound LCIA data and methods; and at to deliver guidance on their use. To initiate the process, UNEP/SETAC convened a Draft Author Team within a defi- nition study to developing guidelines for the development of LCIA, aiming in a first step to:

1) identify user needs for $L C I A$,

2) identify the objectives and deliverables of the LCIA program appropriate to the needs and concerns of all LCA 'stakeholders' and consistent with objectives identified in prior efforts.

The present programme builds on the ISO series of LCA standards, in particular ISO 14042, the LCIA standard, and its related Technical Specification, ISO 14047. Important predecessors of the present programme are the more than ten years of effort by SETAC in advancing LCA, in North America, Asia and Europe. More specifically, the working groups of SETAC Europe on LCIA established a crucial basis for the identification of best available practice in this field. Also, the initiative builds on the ongoing national projects on LCIA, e.g in Japan, Denmark, Holland, Switzerland, USA; and on multinational initiatives such as the OMNIITOX European project. The LCIA Programme complements and strengthens these important international initiatives. It is designed to avoid duplicating their goals and deliverables, addressing the remaining needs.

In order to meet its goals, the team developed, executed, and evaluated a user needs survey in order to enable worldwide participants to make proposals, suggestions and constructive criticism. The user needs survey provided input for setting priorities on both the information and approaches needed for LCIA. Complete results of this survey were included in a final User Needs Analysis report (Stewart and Goedkoop 2003). The report authors also collected input from workshops. This paper summarises the results of this user needs analysis, the update of the LCIA programme aims and briefly describes present task forces working on LCIA. Further publications will describe the new LCIA framework and ongoing work in more detail.

\section{User Need Survey}

\subsection{Description of the user need survey}

The needs analysis provided input to the UNEP SETAC LCIA definition study about issues, categories, values, and priorities for LCIA. The needs analysis was based on responses to 
a survey which was circulated globally. The complete needs analysis report can be found on the UNEP SETAC Life Cycle Initiative's web page; this report details:

- A background to the survey respondents which includes an indication of the regions from which surveys were received, a breakdown of the industrial sectors in which respondents work, details of the departments (marketing, Research and Development etc) in which the respondents work, the manner in which they use LCA, and the confidence which they place in the information delivered by LCA.

- Selection and Prioritisation of Issues in LCIA in which specific issues in LCA were presented and respondents' agreement and/or concerns with these issues gauged.

- Impact Categories to be included in LCA which investigated the perceived significance that respondents placed on both existing and potential new impact categories. This section of the report contrasts differences in perception between the entire respondent group, and those responses received from resource extraction (or non-traditional LCA) countries

- Environmental Values highlighting the end-points which are of concern to the respondents

- Specific Requirements of LCIA that discuss requirements of specific industrial sectors and global regions

- Conclusions including remarks on the potential limitations of the survey results, as well as the expectations that respondents have of UNEP SETAC Life Cycle Initiative.

The intention of this paper is to outline and summarise the information presented in the needs analysis report and to demonstrate how information from respondents to the survey has been used to develop the terms of reference for the next stage of the UNEP SETAC LCIA project. This illustrates the manner in which the needs of the broader LCA are being addressed and demonstrates the transparency of the process.

\subsection{Respondents provenance}

Table 1 details the breakdown of the regions from which the 91 completed surveys were received.

The survey results are obviously be dominated by responses received from Europe. This reflects to some extent the fact that a significant amount of global LCA activity is centred in Europe. Notable in their absence are responses from Japan.

Table 1: Details of regions from which surveys were received

\begin{tabular}{l|c}
\hline UN Region & Number of Respondents \\
\hline Africa & 5 \\
\hline Asia and the Pacific & 7 \\
\hline Europe & 61 \\
\hline Latin America and the Caribbean & 5 \\
\hline North America & 11 \\
\hline Not specified & 2 \\
\hline Total & $\mathbf{9 1}$ \\
\hline
\end{tabular}

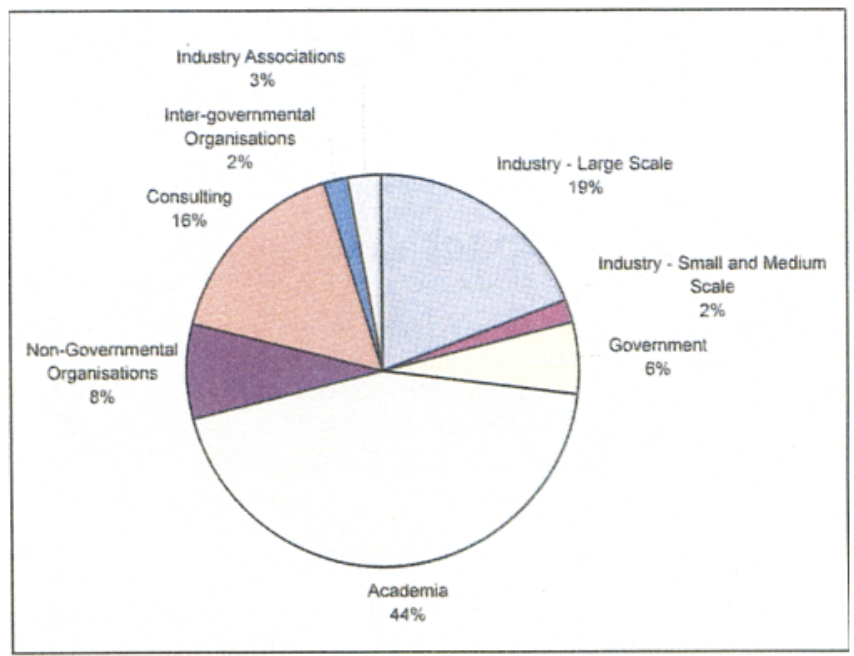

Fig. 1: Breakdown of sectors in which respondents work

Fig. 1 gives an indication of the sectors in which respondents work. Survey results are dominated by those received from academia; however, the spread of sectors from which responses were received is relatively wide.

In order to avoid results being biased by the Europe or Academia dominating participation, answers were analysed separately for non-traditional LCA regions and for nonacademia respondents and compared with all respondents.

\subsection{How is LCA information used?}

Fig. 2 has been included to highlight the manner in which the respondents use LCA information. This information has the potential to inform the link between the LCI, LCIA and LCM elements of the UNEP SETAC Life Cycle Initiative. Fig. 2 demonstrates that, aside from developing LCA software, the majority of respondents either conduct LCAs in order to deliver a baseline assessment of a system, or use LCAs to support decision making processes. The largest class of decisions supported using LCA information are industry decisions.

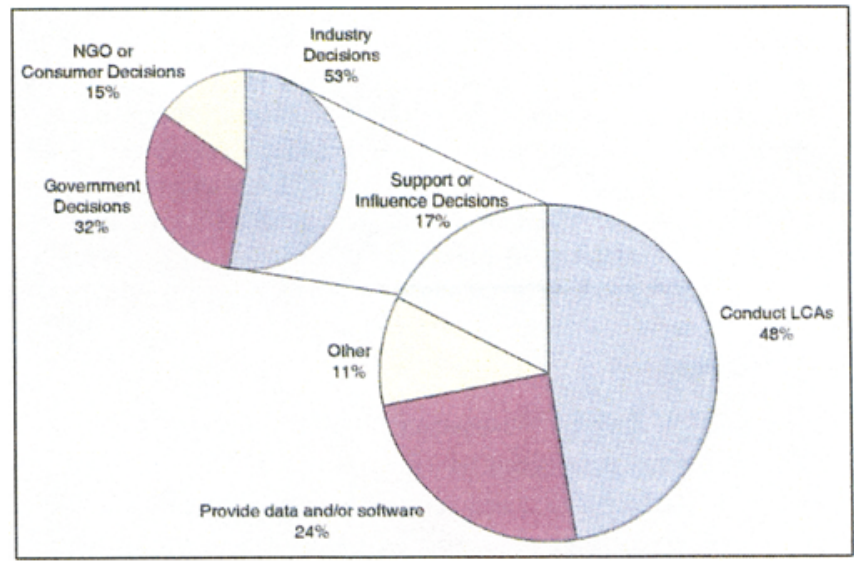

Fig. 2: Breakdown of how respondents use LCA, with further resolution on decisions supported 


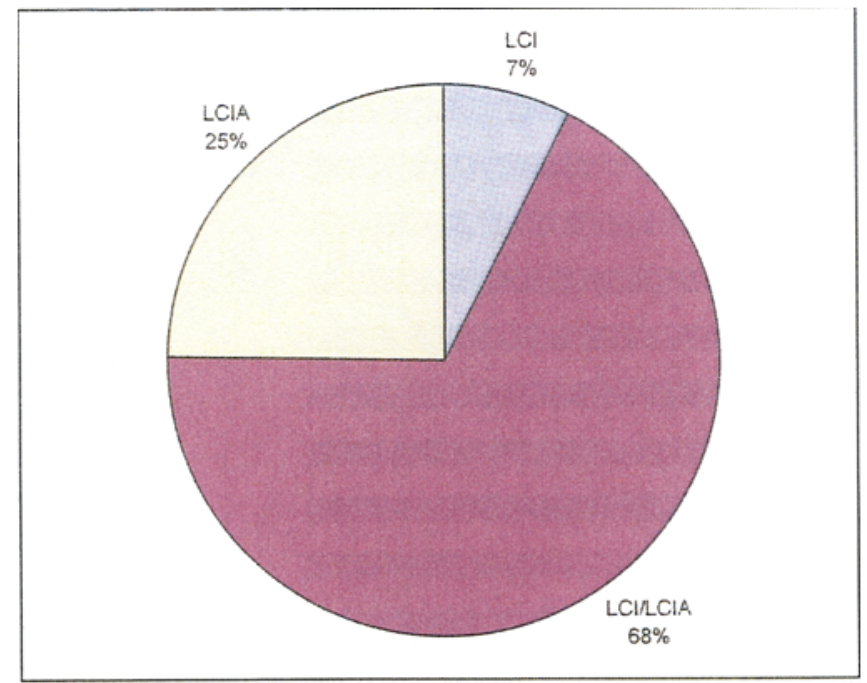

Fig.3: Stages of LCA used to inform decision making by respondents

Respondents were also requested to highlight the stage of LCA which most often informs their decision making. This information is included in Fig. 3. This information should be used in integrating planning between the LCI, LCIA and LCM elements of the UNEP SETAC Life Cycle Initiative. Fig. 3 demonstrates that a significant majority of the respondents use a combination of LCI and LCIA in their work. This reinforces the need to ensure that all elements of UNEP SETAC Life Cycle Initiative are adequately integrated.

Respondents also described their perception of the credibility of LCA methodology. Fig. 4 details these perceptions. Comments offered included the view that, while LCA is wellregarded by governments and NGOs whilst this is not necessarily true for industry. This is particularly the case for the LCIA element of LCA. A further comment highlighted the fact that small distinctions may exist between many similar products, and that in these cases differentiation in the absence of uncertainty insights can be very misleading. Build-

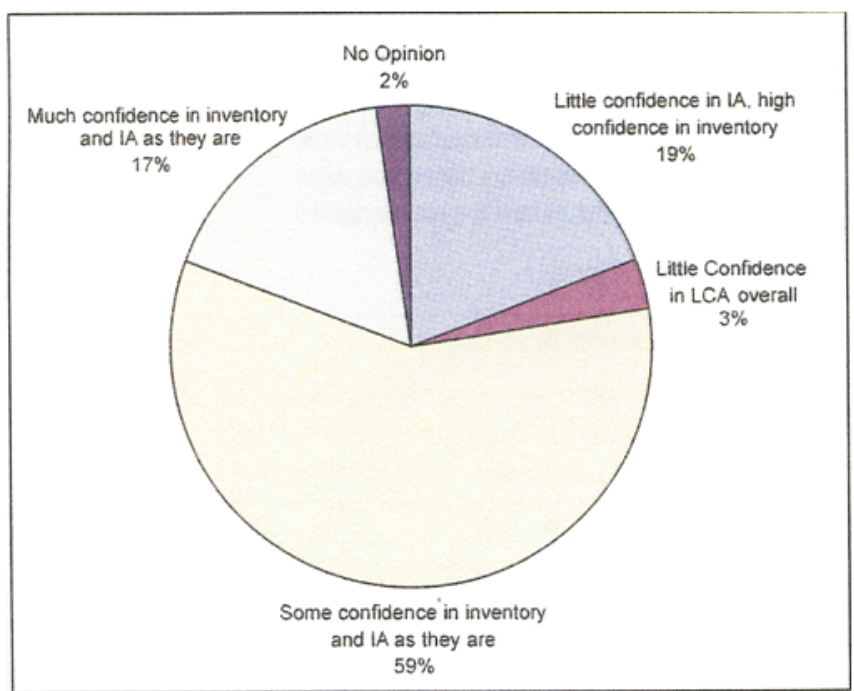

Fig. 4: Details of respondents' confidence in LCI and LCIA information. IA refers to the Impact Assessment element of LCA ing tools with a specific focus, such as on building materials or waste management, can help ensure a degree of robustness/completeness in the studies. In addition, comments were received to the effect that a thorough knowledge of the methodology renders more confidence in the methodology. Fig. 4 demonstrates that, while these users of LCA have some confidence in the outputs of LCA, the level of this confidence is not necessarily high.

\subsection{Selection and prioritisation of issues in LCIA}

Survey respondents were given a list of recognised issues in LCIA and were requested to state whether a) they agreed with the issue as stated in the survey; and b) propose the priority for addressing the issue ${ }^{1}$.

Respondents were asked to report their agreement with these issues (Fig. 5). In this figure, a score of 0 is consistent with complete disagreement with the issue as stated, and a score of 4 is complete agreement with the issue as stated. This information is used to clarify the wording of each issue identified for the LCIA element of UNEP SETAC Life Cycle Initiative as well as in determining whether the issue as presented is of concern to the respondents.

The issues requiring immediate attention within the UNEP SETAC Life Cycle Initiative are:

- Need for transparency in the methodology

- Need for Scientific confidence

- Need for Scientific co-operation

- A recommended set of factors and methodologies should be developed

Next in order of priority, is the need to develop an adaptive framework compatible with other dimensions of sustainability, easily interpretable indicators provide recommended factors and guidance for weighting enabling an improved ease of use. The development of models with spatial and temporal differentiation and higher level of information details were also perceived to be important by a significant number of the respondents.

Additional comments from the survey respondents included specific reference to information uncertainty, the need for consistency in the application of LCA, and the potential need for the development of a methodology for screening new/ proposed LCIA impact categories.

\subsection{Impact categories to be included in LCA}

Survey respondents were requested to list those impact categories which ${ }^{2}$ :

- should be 'Required' as part of the minimum set for a credible method, or

- would be nice to include or 'Nice to Know', or

- are of 'Low Priority' and could be left out

Fig. 6 includes the information recorded for this question.

\footnotetext{
1 A further option of 'No Opinion' was included to ensure that information gathered was not biased by scurrilous data

2 Again a 'No Opinion' option was included
} 


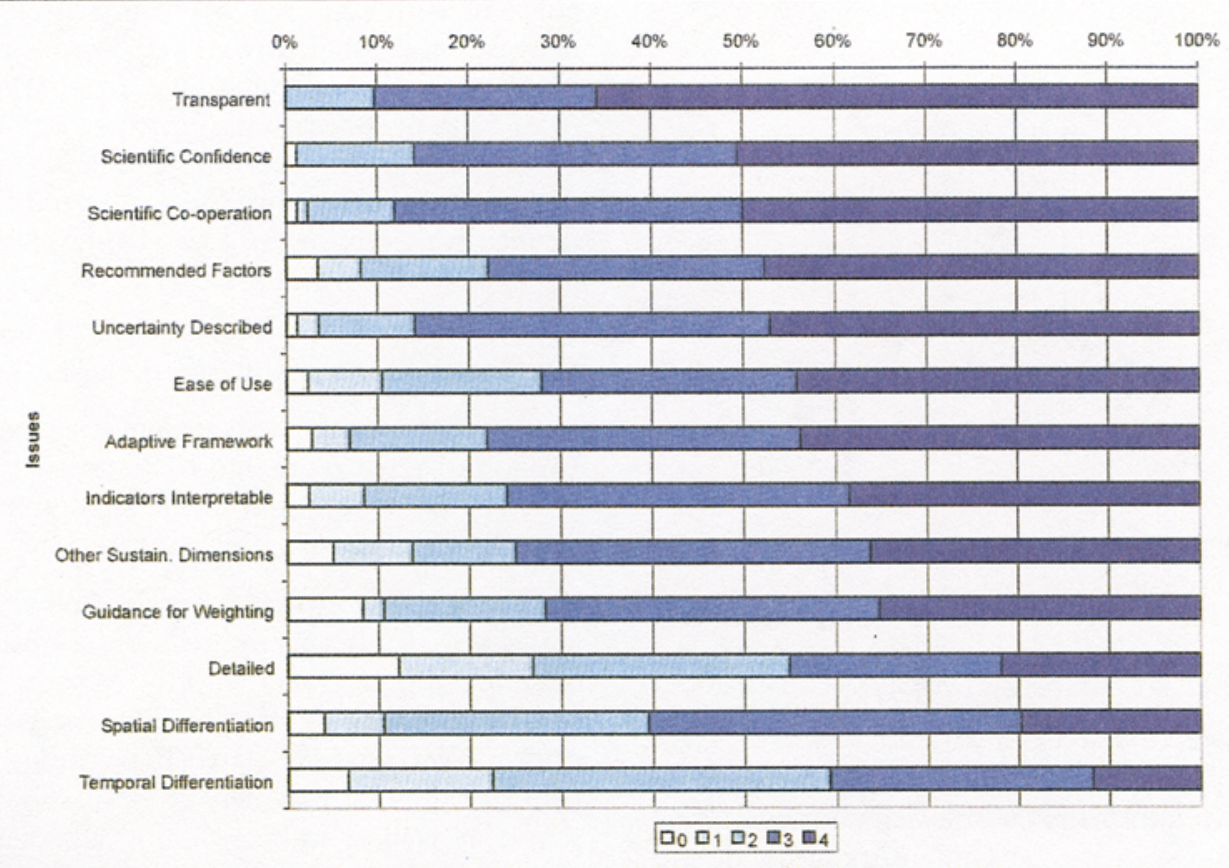

Fig. 5: Agreement with the stated issues in the survey: a score of 0 expresses complete disagreement and a score of 4 complete agreement with the issue as stated

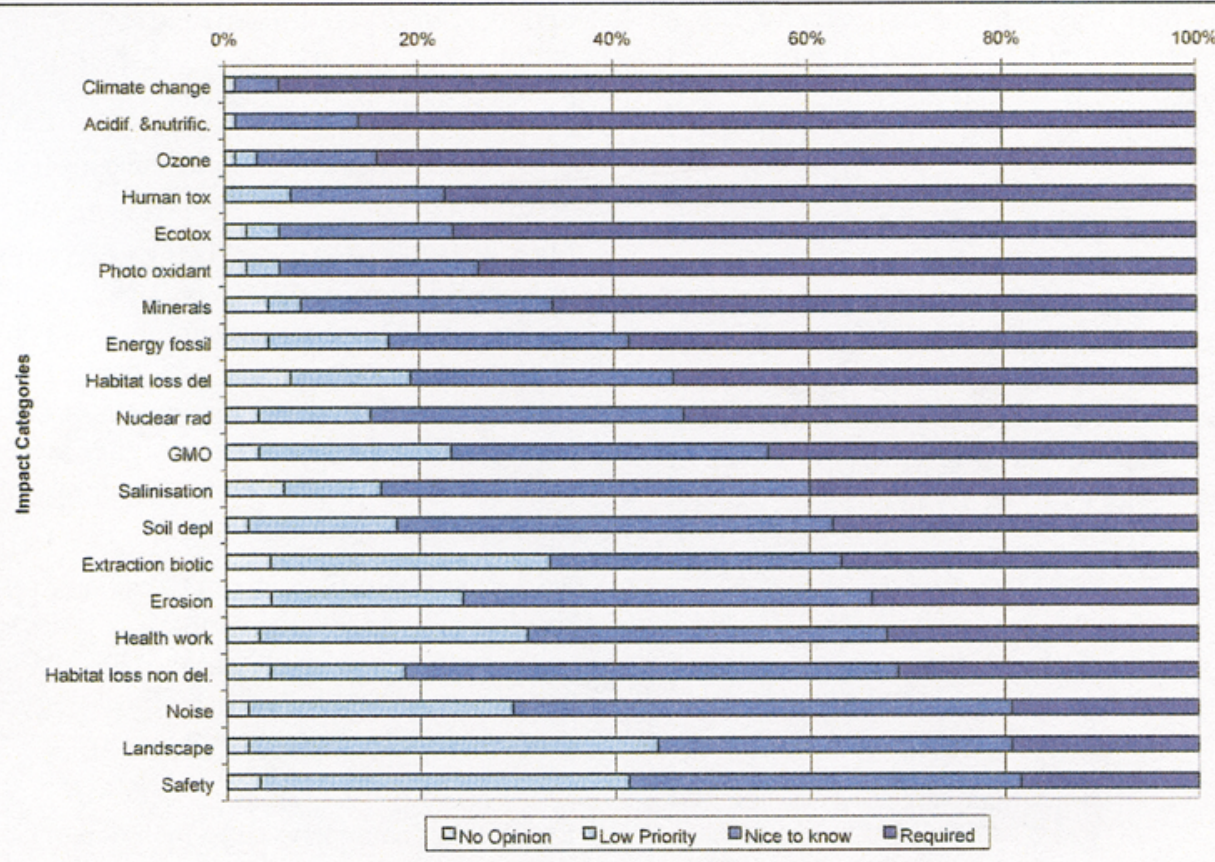

Fig. 6: Importance of impact categories included in survey

In addition, respondents were requested to specify additional impact categories which they require to be included in LCA. These additional categories are included in the table below.
While it might have been possible to base the conclusions of the needs analysis on the aggregated set of information included above, one of the stated objectives of the UNEP

\begin{tabular}{|l|l|}
\hline Required & Nice to Know \\
\hline - PAF & $\begin{array}{l}\text { - Manpower and qualification in the work environment } \\
\text { - Humanity issues related to ILO Conventions in the work } \\
\text { - Use of scarce resources should be taken into account }\end{array}$ \\
$\begin{array}{l}\text { - Some indicator of endogenous groups that might be present in study areas } \\
\text { - Water pollution, water usage (significant focus for a number of respondents) } \\
\text { - Landuse incorporating a consideration of land lost through sealing and destruction } \\
\text { - Indication of accumulation within systems (assuming that a modelling methodology exists) } \\
\text { - Risk of accidents }\end{array}$ & $\begin{array}{l}\text { - Odour } \\
\text { - Dessication }\end{array}$ \\
\hline
\end{tabular}




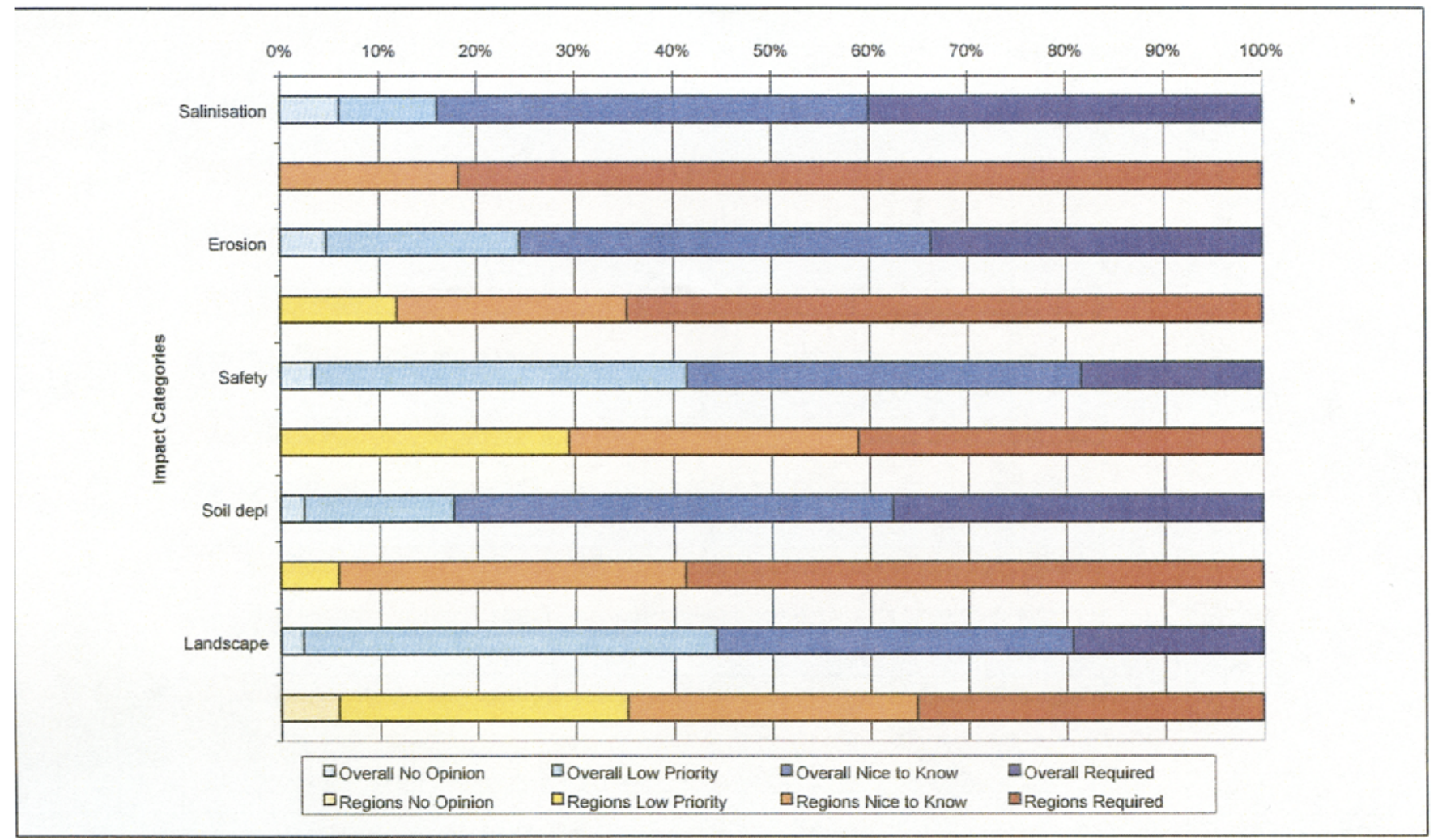

Fig. 7: Issues of specific concern to regions previously not engaged with the development of LCA (red) compared to concerns in the overall user need assessment (blue)

SETAC Life Cycle initiative is to ensure that the needs of regions not previously engaged in the formulation and development of LCA are adequately addressed. For this reason the responses received from Africa; Latin America and the Caribbean; and Asia and the Pacific have been contrasted with the overall set of responses. In developing Fig. 7, only those impact categories where the difference in the sum of scores for 'Required' and 'Nice to Know' between the overall survey respondents and the regional survey respondents was greater than $10 \%$ have been included. These impact categories have been arranged according to the magnitude of the differences in scores. Fig. 7 demonstrates that the concerns of these regions do differ quite significantly from those expressed by the overall survey respondents.

From this information the following conclusions can be drawn:

- In order to meet the needs of those regions who have not previously been involved in development of LCA, attention should be paid to water usage, erosion, salination, landscape destruction and habitat losses. The most significant difference between the overall results and the region specific results were recorded for salination and erosion.

- These regions are also asking for focus to be placed on occupational health and safety issues. However, this should be reviewed in the light of the general comments received about LCA not being the correct tool with which to assess these effects.

Fig. 1 demonstrated that the survey responses are dominated by those from academia. In order to determine whether this has skewed the results presented in this section a similar process was conducted for non-academia respondents as has been described above for the non-traditional LCA regions. Fig. 8 demonstrates that there is limited difference in perception of significance of impact categories between academic and non-academic users of LCA (differences lower than $15 \%$ ). Attention should be paid to these results in establishing the action plan for the LCIA element of UNEP SETAC Life Cycle Initiative as this describes the requirements of users other than academia.

Table 2 has been constructed to guide where emphasis might be placed in impact category development. In constructing this table:

- those impact categories which scored more than $50 \%$ Required are placed in the 'Required' column,

- those impact categories which scored $70 \%$ when Required and Nice to Know are added in the 'Nice to Know' column,

- the balance of the impact categories being assigned a low priority ${ }^{3}$.

\subsection{Outlook of the user need assessment}

The User Needs Assessment delivered an understanding of how and why user gather LCA information and highlighted information gaps in LCA. Further, required, nice to know and low priority impact categories were highlighted. Significant differences between the requirements of different global regions were noted. These will be addressed through the continuing work of the LCIA programme.

${ }^{3}$ These results have been formulated using the overall and not the region specific results 


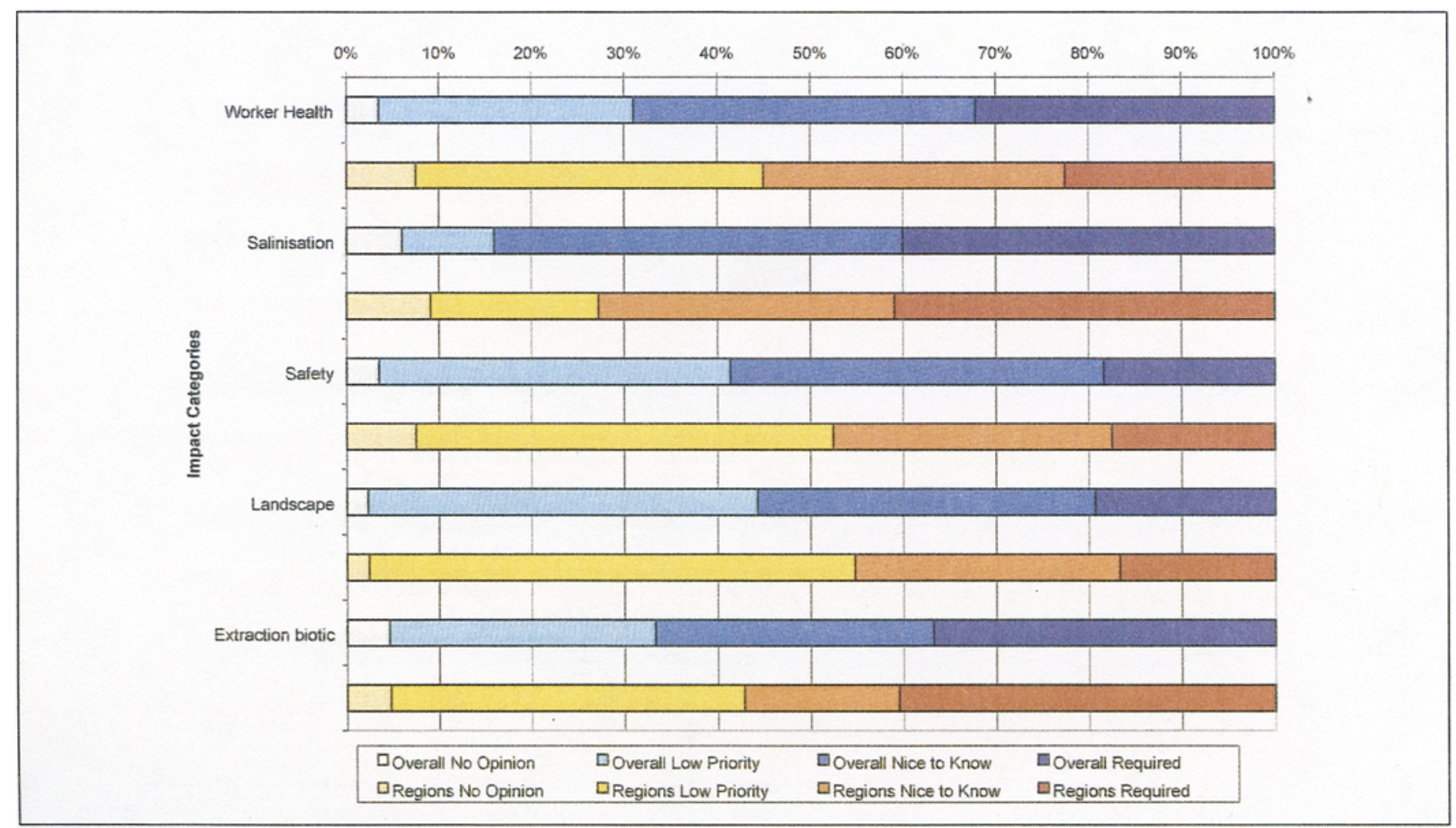

Fig. 8: Issues of specific concem to non-academic respondents (red) compared toconcerns in the overall user need assessment (blue)

Table 2: Significance of impact categories. 'Required column': more than $50 \%$ Required; impact categories which scored $70 \%$ when 'Required' and 'Nice to Know' are added in the 'Nice to Know' column

\begin{tabular}{l|l|l}
\hline Required & Nice to Know & Low Priority \\
\hline - Climate Change & - Salinisation & \\
- Ozone Depletion & - Erosion & \\
- Habitat loss as result of deliberate actions & - Soil Depletion & \\
- Human toxicity & - Habitat loss as a resull of indirect actions & - Health of workers \\
- Eco-toxicity & - Noise & - Safety \\
- Acidification and Eutrophication & - Use of GMOs & \\
- Photo-oxidants & & \\
- Extraction of Minerals & & \\
- Energy from Fossil Fuels & & \\
- Nuclear Radiation & & \\
- Water usage & & \\
\hline
\end{tabular}

${ }^{1}$ Not in the initial list, but highlighted as being of significance through the needs analysis

${ }^{2}$ Classified as 'Required' if only answers from non-traditional LCA countries are considered

${ }^{3}$ Classified as 'Nice to Know' if only answers from non-traditional LCA countries are considered

\section{Inputs from Different Workshops}

As a complement to the user need survey, different workshops were organised by, or in collaboration with, the Life Cycle Initiative (Jolliet 2003) to enable direct inputs from a broad range of participants from different continents to the definition of the LCIA work programme:

- Organised in collaboration with the mining industry (ICMM) and APEC (Asia-Pacific economies), the Montreal and Apeldoorn workshops brought together specialists from different fields related to minerals and metals, to discuss how to improve the relevance of LCA (including LCIA) in this field (15 to 17 April 2002 and 15 March 2004). These workshops identified metals-specific research issues that need to be investigated to improve current approaches. They also showed the need to guide present LCA users on both the potential, and the limitations, for the application of present LCA methodologies to metals.

- The Vienna (16 May 2002) and Hamburg workshops focused on the establishment of a flexible LCIA framework, including midpoint and damage indicators. They investigated new possibilities to reconcile approaches based on 
classical impact category indicators (sometimes called midpoint) and damage oriented approaches (sometimes called endpoint). They showed that a) the level of detail in recommended LCIA methods should be restricted to what is really necessary in order to avoid the introduction of debatable statements, b) it is always useful to try to model up to endpoint or damages, but it is essential to quantify and specify related uncertainties, c) case studies are needed to test new indicators, method feasibility, etc., d) the taxonomy structure proposed by US-EPA is an interesting structure which deserves further development, e) the framework and list of categories proposed in the definition study of the LC Initiative can now be considered as a final first draft, and f) there is a significant interest from several participants to be involved in a group investigating further how social and economical dimensions should be integrated into the LCIA framework.

- The Tsukuba Workshop was organised by AIST, it was titled 'Gateway to Life Cycle Impact Assessment for APEC Member Economies' (7 November 2002) and showed that there is some convergence between damage and midpoint impact modelling and further that there is potential to adapt much of what has been done in Europe for countries in the APEC region, possibly based on environmental 'archetypes' rather than strict geographical areas.

- At the Barcelona LCIA workshop (December 2002) the main proposal and preliminary framework of the Draft Author Team was presented to a larger audience including industry partners. This workshop provided interesting feedback. The discussion on the preliminary framework clearly shows two main points of view:

- On the one hand, several users emphasise the need to stay at midpoint level, further developments and methodological improvement being clearly needed at that level (a midpoint level approach was not considered old fashioned). There is also a risk of reducing user confidence if methods with significant uncertainties are recommended.

- On the other hand, some users emphasise an interest in damage assessment, e.g for communicating to governments, for understanding relevance or answering industrial needs. The survey reveals that these two points of view do not occur preferentially among academia or industry, but are held almost evenly between the groups.

Thus, the proposed framework should enable the delivery of results at both midpoint and damage levels in a consistent way, a quantitative approach to damage assessment being recommended only if additional uncertainty is not too high. The proposed framework has to be flexible enough to enable the incorporation of new categories or of different value systems without major change. However, it has to be rigid enough to enable a consistent structuring of the way impact categories will be described.

\section{Objectives of the Life Cycle Impact Assessment Programme}

The LCIA programme aims at the enhancement of the availability of sound LCA data and methods, and at guidance on their use.
The guidelines for the life-cycle initiative are based on the recognition that the scientific development of LCIA is ongoing, must avoid establishing one very specific method or dataset, and must stimulate innovation. On the other hand, any guidance developed must discourage a situation in which 'anything goes', as this can lead to confusion and misuse of the methodology. Therefore guidance and recommendations are needed. Because the ISO 14042 framework leaves room for a very wide variety of approaches and interpretations, the application of LCIA is rather difficult for non-experts to interpret or apply. Furthermore, since the publication of the ISO standard, new developments such as the concept of Midpoint and Endpoint approaches have emerged. These methods will be better explained and better integrated into the existing methodology.

In parallel, the LCIA programme will stimulate input from specialists in different fields into LCA, at least for review work; and bring scientist and industrialists to collaborate closer in addressing practical and scientific challenges, leading to better quality and easier application of LCIA methods.

In this context and according to the user needs survey, the LCIA programme aims to:

- Provide guidance and recommendations on LCIA and make methodologies widely available for users through the creation of a worldwide accessible information system. Until recommended factors and models are defined, the information system will provide links to existing methods and characterization factors at midpoint and damage levels, enabling users to easily access state-ofthe-art methods and to fill-in relevant elements of the LCIA framework. Once recommended methods have been ascertained, the initiative will lay out a clear process for revising the framework, e.g. on a triannual basis.

- Develop a consistent conceptual framework for LCIA, including the relationships with the LCI results on the one hand, and damages on the other hand. This framework will describe how impact pathways (composed of environmental processes) link the LCI results to midpoint and damage indicator(s), based on impact pathways. Preferably this framework will be the same for both midpoint and damage approaches. The initiative leaves it up to the user to decide whether midpoint indicators, damage indicators, or even LCI results are used as the basis for decision making.

- Establish recommended characterisation factors and related methodologies for different impact categories, possibly consisting of sets at midpoint and at damage level, including new impact categories for developing countries. Models of impact pathways used to calculate characterisation factors will be recommended for the different impact categories. Specific attention will be paid to ensuring that these models are transparent and that the assumptions and model choices are adequately documented. The initiative intends to describe assumptions and choices and give recommendations on how to manage and document these. This will provide guidance to 
both users and model developers on how to increase consistency and transparency in LCIA. These factors will cover a broad range of LCI results. 'Generic situation dependency' can be introduced to account for those main archetypical situations leading to important variations in characterisation factors and therefore justifying a differentiation within the quantification of LCIA results. These will be incorporated in an LCIA database to facilitate application by users.

- Stimulate collaboration between scientists and industrialists. The LCIA programme will stimulate input from specialists in different fields into LCA, at least for review work, and bring scientists and industrialists to collaborate closer in addressing practical and scientific challenges, leading to better quality and easier application of LCIA methods.

In addition, the Life Cycle Initiative should provide a library of case studies illustrating good/successful and bad/unsuccessful uses of LCA methodologies in practice, including LCIA and the two other UNEP/SETAC programmes on Life Cycle Inventory (LCI) and Life cycle Management (LCM).

\section{Present Work and Perspectives}

The work of the LCIA programme of the UNEP/SETAC Life Cycle Initiative has started within four task forces that are described in more detail on the UNEP/SETAC website and will be described in future publications in this journal. In short, work is being carried out according to the following tasks and aims (see http://www.uneptie.org/pc/sustain/ lcinitiative/lcia program.htm for more details):

- Task Force 1 on 'LCIA Information system and framework' aims on the one hand to develop an LCLA information system providing guidance on LCIA (http://www. uneptie.org/pc/sustain/lcinitiative/lcia program.htm). This system presently provides access to latest/most widely used LCIA methods and characterization factors, including hyperlinks to their respective home websites. The developers of methods not yet included on this site are invited to contribute information on their methods. This information should be provided on a template which is available on request. Methods will only be included on this web-site under the condition that the method is freely available, transparent and documented. Further, task force 1 aims to develop a general LCIA framework enabling assessment both at midpoint and at damage levels in a consistent way.

- Task Force 2 'Natural resources and land use' aims to establish recommended practice and guidance for natural resources and land use categories. This resource impact category is especially crucial for developing countries, in which the development of related impact categories such as water use, salinisation, dessication and erosion is essential to contribute to avoiding impacts relevant to these regions.

- Task Force 3 on 'Toxic impacts' aims to establish recommended practice and guidance for use for the ecotoxicity, human toxicity and related categories with direct effects on human health, i.e: ecotoxicity, human toxicity, ionising radiation, accidents and noise. Photochemical smog and respiratory inorganics was coordinated with task force 4 (as described below). At the Review workshop (1 and 2 December 2003) a matrix structure was proposed as a flexible framework for Life Cycle Toxicity Assessment. This will be the base upon which to establish libraries of processes and matrix factors of substance data and estimation tools and of geographic data (landscape data etc.).

- Task Force 4 on 'Transboundary impacts' aims to establish recommended practice and guidance for use in transboundary categories, i.e: climate change, ozone depletion, aquatic and terrestrial eutrophication and acidification. Photooxidant formation and respiratory inorganics (Primary and secondary particles) will be coordinated with task force 3. A point of focus is the need for adapting knowledge from other scientific communities focusing on environmental modelling to the assessment of Life Cycle Impacts linked to functions and products.

All participants willing to contribute to these efforts are invited to contact the LCIA programme manager (olivier.jolliet@epfl.ch) or the different task force leaders. You are also invited to join the next LCIA workgroup meeting that will take place in at the world SETAC congress in Portland on Thursday 18 November 2004.

This next SETAC world congress has been formulated as a special workshop on 'Sustainable Production, Use and Recycling of Natural Resources', with sessions on 'depletion impacts associated with resource extraction', 'land use' and on 'comparative risk studies and LCIA toxicity impacts' (http://www.uneptie.org/pc/sustain/lcinitiative/Symposium\% 20Portland.htm). The submission of abstracts before 31 May 2004 is greatly welcomed (http://www.setac.org/portland.html).

Further publications in Int J LCA will describe the new LCIA framework and the ongoing work in more detail.

\section{References}

Jolliet O, Brent A, Goedkoop M, Itsubo N, Mueller-Wenk R, Pena C, Schenk R, Stewart M, Weidema B, Bare J, Heijungs R, Pennington D, Rebitzer G, Suppen N, Udo de Haes H (2003): Final Report of the LCIA Definition Study. Life Cycle Impact Assessment Programme of the UNEP/SETAC Life Cycle Initiative, 12/2003. Download at: <http://www.uneptie.org/pc/sustain/ Icinitiative/lcia program.htm>

Jolliet O (2003): Overview of the LCIA work meeting results in Vienna, Tsukuba and Barcelona. Life Cycle Impact Assessment definition study: Background document II Download at: $<\underline{\text { http:l }}$ /www.uneptie.org/pc/sustain/lcinitiative/lcia program.htm>

Stewart M, Goedkoop M (2003): LCIA Needs analysis report of the UNEP-SETAC Life Cycle Initiative. Life Cycle Impact Assessment definition study:

Background document I. Download at: <http://www.uneptie.org/ pc/sustain/lcinitiative/lcia program.htm> 